\title{
Shielding properties of a conducting bar calculated with a boundary integral method
}

\author{
L. O. Fichte, S. Lange, Th. Steinmetz, and M. Clemens
}

Professur für Theoretische Elektrotechnik und Numerische Feldberechnung, Helmut-Schmidt-Universität, Universität der Bundeswehr Hamburg, PO. Box 700822, 22008 Hamburg, Germany

\begin{abstract}
A plane rectangular bar of conducting and permeable material is placed in an external low-frequency magnetic field. The shielding properties of this object are investigated by solving the given plane eddy current problem for the vector potential with the boundary integral equation method. The vector potential inside the rectangle is governed by Helmholtz' equation, which in our case is solved by separation. The solution is inserted into the remaining boundary integral equation for the exterior vector potential in the domain surrounding the bar. By expressing its logarithmic kernel as a Fourier integral the overall solution inside and outside the bar is calculated using analytical means only.
\end{abstract}

\section{Introduction}

Subject of this investigation are the shielding properties of a rectangular bar of constant conductivity $\kappa_{i}$ and permeability $\mu_{i}$. The space outside the bar has the arbitrary constant permeability $\mu_{a}$ and conductivity $\kappa_{a}=0$. The vector $\boldsymbol{r}_{q}=x_{q} \boldsymbol{e}_{x}+y_{q} \boldsymbol{e}_{y}$ is pointing at the lower left corner of the bar, the dimensions of it being $a$ and $b$, as shown in Fig. 1. We name the cross-section of the bar $\Omega$ and its contour $C=\partial \Omega$.

An exciting loop consists of two thin conductors, which carry the currents $i(t)$ and $-i(t)$, with $i(t)=\Re\left\{\underline{I} e^{j \omega t}\right\}, \underline{I}$ being the phasor describing the complex current. They are located at

$\boldsymbol{r}_{\mathrm{e}}= \pm x_{e} \boldsymbol{e}_{x}$.

The angular frequency $\omega$ is considered to be low enough so that displacement currents can be neglected: $\boldsymbol{J} \gg \frac{\partial}{\partial t} \boldsymbol{D}$, where $\boldsymbol{J}$ is the current density inside the bar and $\boldsymbol{D}$ the electric flux.

The $z$-directed dimensions of both bar and loop are supposed to be infinite.

\section{Differential equations for the vector potential}

As all exciting currents are oscillating at one single frequency, all fields will show the same time dependency and can be expressed by their complex phasors.

Correspondence to: $\mathrm{L}$. O. Fichte

(lars-ole.fichte@unibw-hamburg.de)
Introducing a complex vector potential $\boldsymbol{A}$ as $\boldsymbol{B}=\operatorname{curl} \boldsymbol{A}$, where $\boldsymbol{B}$ denotes the magnetic flux, and investigating its properties, we find that an additional term has to be added to the vector potential if we consider points inside the bar. The additional term can be expressed as the time domain integral of the gradient of a complex electrical scalar potential $\phi_{i}(z)$ by

$\int\left(\operatorname{grad} \phi_{i}\right) \mathrm{d} t=C \boldsymbol{e}_{z}$,

where $C$ denotes an unknown complex constant. The vector potential $\boldsymbol{A}_{\mathrm{i}}$ inside the bar can be redefined by using the Buchholz convention

$A_{i}^{*}=A_{i}+\int \operatorname{grad} \Phi_{i} \mathrm{~d} t=A_{i}+C \boldsymbol{e}_{z}$

from which the fields can be computed with

$\boldsymbol{B}_{\mathrm{i}}=\operatorname{curl} \boldsymbol{A}_{\mathrm{i}}^{*} \quad$ and $\quad \boldsymbol{E}_{\mathrm{i}}=-j \omega \boldsymbol{A}_{\mathrm{i}}^{*}$.

From Maxwell's equations we find that the modified vector potential inside the bar is governed by Helmholtz' equation, which for complex fields takes the form

$\Delta \boldsymbol{A}_{\mathrm{i}}^{*}-j \omega \mu_{i} \kappa_{i} \boldsymbol{A}_{\mathrm{i}}^{*}=0$.

Outside the bar the equation

$\Delta \boldsymbol{A}_{\mathrm{a}}=-\mu_{a} \boldsymbol{J}_{e}$,

is valid for the vector potential $\boldsymbol{A}_{\mathrm{a}}$. The exciting current density $\boldsymbol{J}_{e}$ can be expressed as

$\boldsymbol{J}_{e}=\left\{\delta_{1}\left(x-x_{e}\right)-\delta_{1}\left(x+x_{e}\right)\right\} \delta_{1}(y) \cdot \underline{\boldsymbol{I}} \boldsymbol{e}_{z}$.

The symbol $\delta_{1}\left(x-x_{o}\right)$ denotes the one-dimensional Dirac function; accordingly, $\delta_{2}$ and $\delta_{3}$ are the two- and threedimensional Dirac functions.

Since we allow only $z$-directed exciting currents, all vector potentials will also be exclusively $z$-directed. They can be described by their $z$-components $A_{\mathrm{az}}$ and $A_{\mathrm{iz}}^{*}$, respectively. The boundary conditions for $A_{\mathrm{az}}$ and $A_{\mathrm{iz}}^{*}$ are

$A_{\mathrm{az}}=A_{\mathrm{iz}}^{*}-C \wedge \frac{1}{\mu_{a}} \boldsymbol{n}_{o} \cdot \operatorname{grad} A_{\mathrm{az}}=\frac{1}{\mu_{i}} \boldsymbol{n}_{o} \cdot \operatorname{grad} A_{\mathrm{iz}}^{*}$,

where $\boldsymbol{n}_{o}$ is a unit vector normal to the boundary. 


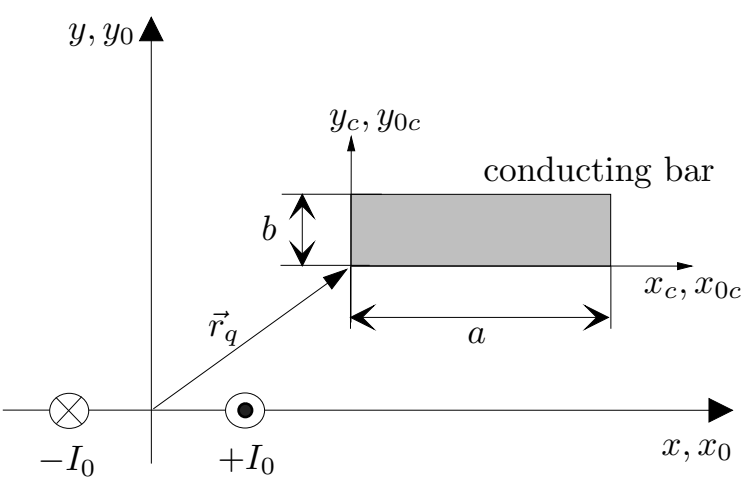

Fig. 1. Screening bar and exciting loop.

\section{Derivation of Boundary Integral Equation (BIE)}

In Green's second theorem

$$
\begin{aligned}
& \iiint_{\tau}(\Phi \Delta \Psi-\Psi \Delta \Phi) \mathrm{d} \tau= \\
& =\underset{F=\partial \tau}{\mathrm{v}}(\Phi \operatorname{grad} \Psi-\Psi \operatorname{grad} \Phi) \cdot \boldsymbol{n}_{\boldsymbol{o}} \mathrm{d} F,
\end{aligned}
$$

we insert the single component of the vector potential $A_{\mathrm{a}}$ as $\Psi$ and a kernel function $K$ satisfying $\Delta K=-\delta_{3}$ as $\Phi$. As a result, we get an integral representation valid for $A_{\mathrm{a}}$ :

$$
\begin{aligned}
& A_{\mathrm{a}}=-\underset{\substack{\mathrm{V} \\
\mathrm{V} \tau}}{+\infty}\left(A_{\mathrm{a}} \operatorname{grad} K-K \operatorname{grad} A_{\mathrm{a}}\right) \cdot \boldsymbol{n}_{o} \mathrm{~d} F+ \\
& +\left.\mu_{a} \underline{I} \int_{-\infty}^{+\infty} K\right|_{\substack{x_{o}= \pm x_{e} \\
y_{o}=0}} \mathrm{~d} z_{o} .
\end{aligned}
$$

The integrand of the surface integral at the right hand side of the above equation does not depend on $z$. Hence the $z$ directed integration is only affecting the kernel function $K$, for which the elementary Kernel function

$K=\frac{1}{4 \pi r}$,

is inserted. Thereby we can define a new kernel function $G_{20}$ as

$$
\begin{aligned}
G_{20} & =\int_{-\infty}^{+\infty} K \mathrm{~d} z_{o}=\frac{1}{4 \pi} \int_{-\infty}^{+\infty} \frac{\mathrm{d} z_{o}}{\sqrt{\left(x-x_{o}\right)^{2}+\left(y-y_{o}\right)^{2}+\left(z-z_{o}\right)^{2}}} \\
& =-\frac{1}{2 \pi} \ln \left(\frac{\sqrt{\left(x-x_{o}\right)^{2}+\left(y-y_{o}\right)^{2}}}{\rho_{o}}\right)=-\frac{1}{2 \pi} \ln \left(\frac{\rho}{\rho_{o}}\right),
\end{aligned}
$$

with $\rho=\sqrt{\left(x-x_{o}\right)^{2}+\left(y-y_{o}\right)^{2}}$ and $\rho_{o}=$ const. While we are aware that the above integral is divergent, it can be solved by performing a suitable renormalization.

The function $G_{20}$ satisfies $\Delta G_{20}=-\delta_{2}$, as is shown in Ehrich et al. (2000).

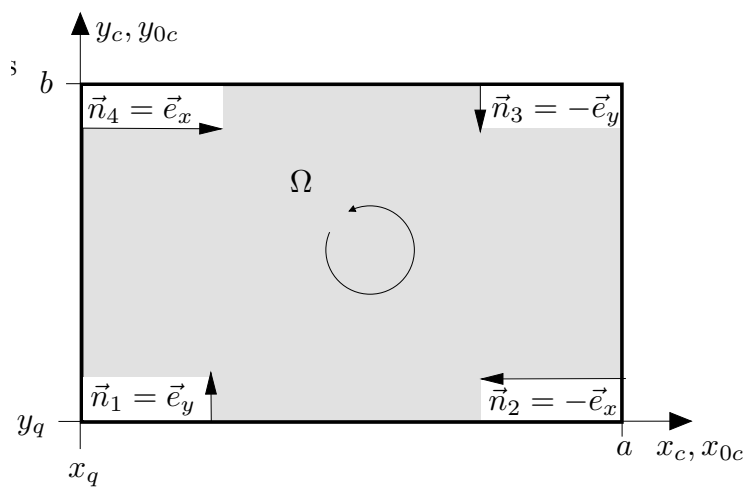

Fig. 2. Normals on boundary scetions.

If we use the new kernel and define the influence of the exciting loop as

$A_{\mathrm{e}}:=\left.\mu_{a} \underline{I} G_{20}\right|_{\substack{x_{o}= \pm x_{e} \\ y_{o}=0}}$,

we can write the integral representation for $A_{a}$ as

$A_{\mathrm{a}}=-\oint_{C=\partial \Omega}\left(A_{\mathrm{a}} \operatorname{grad} G_{20}-G_{20} \operatorname{grad} A_{\mathrm{a}}\right) \cdot \boldsymbol{n}_{o} \mathrm{~d} s+A_{\mathrm{e}}$.

We use the cross-section of the bar as integration domain $\Omega$ with its facet normals defined as shown in Fig. (2).

By taking into account the boundary conditions discussed above, we get an integral representation for the vector potential outside the bar:

$$
\begin{aligned}
& A_{\mathrm{a}}= \\
& -\oint_{C=\partial \Omega}\left[\left(A_{i}^{*}-C\right) \operatorname{grad} G_{20}-G_{20} \frac{\mu_{a}}{\mu_{i}} \operatorname{grad} A_{i}^{*}\right] \cdot \boldsymbol{n}_{o} \mathrm{~d} s+A_{\mathrm{e}} .
\end{aligned}
$$

It can be shown that

$$
\oint_{C=\partial \Omega}\left(C \operatorname{grad} G_{20}\right) \cdot \boldsymbol{n}_{o} \mathrm{~d} s=0,
$$

so we can omit this term in Eq. (3).

In a last step we take the normal derivative of $A_{\mathrm{a}}$ on each of the four contour sections of $\Omega$ and move the observation point to the very same section, i.e.
(1) $y=y_{q}$,
(3) $y=y_{q}+b$,
(2) $x=x_{q}+a$,
(4) $x=x_{q}$.

As a result, we get four independent integral equations

$\frac{\mu_{a}}{\mu_{i}} \boldsymbol{n}_{o} \cdot \operatorname{grad} A_{\mathrm{i}}^{*}=\boldsymbol{n}_{o} \cdot \operatorname{grad}$

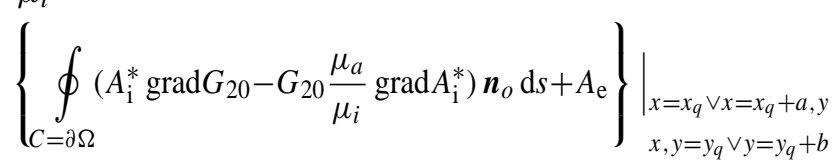

Conventionally, one would derive another boundary integral equation, this one valid for $A_{\mathrm{i}}^{*}$, using

$G_{20 \mathrm{i}}=\frac{1}{4 j} \mathrm{H}_{0}^{1}\left(k \frac{\rho}{\rho_{0}}\right) \quad$ with $\quad k^{2}=j \omega \kappa \mu_{i}$ 


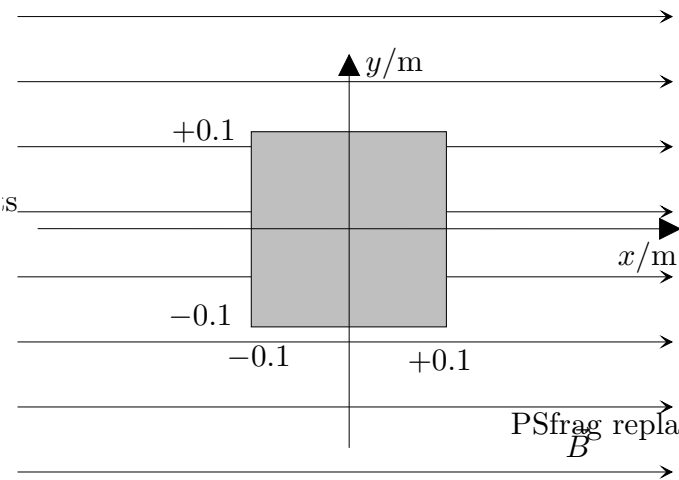

Fig. 3. Quadratic bar in homogenous field.

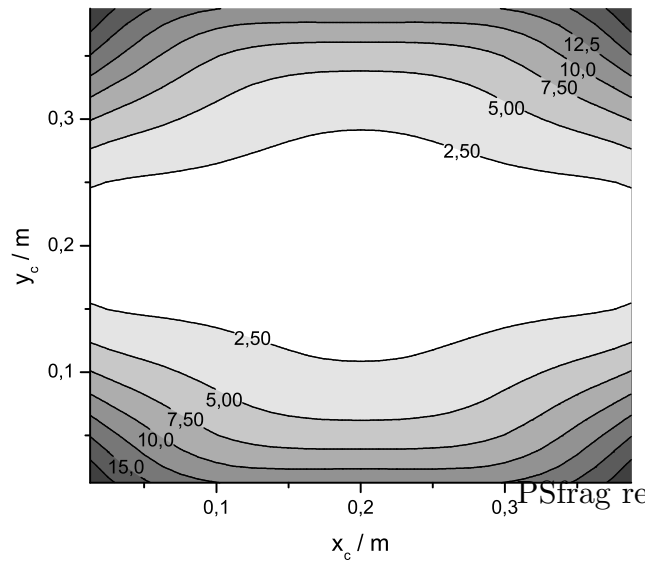

Fig. 4. $\operatorname{Abs}\left(A_{\mathrm{i}}^{*}\right)$ for bar in homogenous field.

as a kernel function Hanson and Yakovlev (2002). The function $\mathrm{H}_{0}^{1}$ is the Hankel function of the first kind and order zero. The resulting system of two integral equations could be solved to yield the solutions for the two unknown functions $A_{\mathrm{a}}$ and $A_{\mathrm{i}}^{*}$.

Here, we find a solution to Eq. (1) by separation, using a product of functions depending only on one coordinate for the descrition of $\boldsymbol{A}_{\mathrm{i}}^{*}$.

$A_{\mathrm{i}}^{*}\left(x_{c}, y_{c}\right)=$

$\sum_{n=1}^{\infty}\left[v_{1 n} \cosh \left(\beta_{n}\left(b-y_{c}\right)\right)+v_{3 n} \cosh \left(\beta_{n} y_{c}\right)\right] \cos \left(\alpha_{n} x_{c}\right)$

$+\left[v_{2 n} \cosh \left(\tilde{\beta}_{n}\left(a-x_{c}\right)\right)+v_{4 n} \cosh \left(\tilde{\beta}_{n} x_{c}\right)\right] \cos \left(\tilde{\alpha}_{n} y_{c}\right)$,

$\alpha_{n}=\frac{n \pi}{a}, \beta_{n}=\sqrt{\alpha_{n}^{2}+j \omega \kappa \mu}$,

$\tilde{\alpha}_{n}=\frac{n \pi}{b}, \tilde{\beta}_{n}=\sqrt{\tilde{\alpha}_{n}^{2}+j \omega \kappa \mu}$.

Then, the unknown constants $v_{i n}, i=1, \ldots, 4$ will have to be determined.

We can now insert the result for $A_{\mathrm{i}}^{*}$ into Eq. (4). Using the

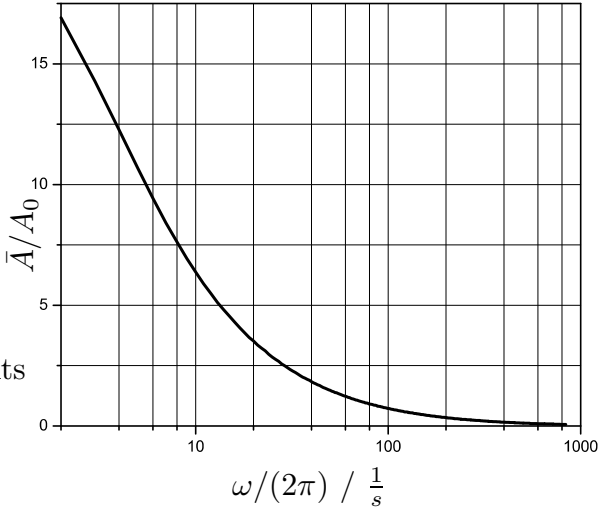

Fig. 5. $\bar{A} / A_{0}$ as a function of $\omega, \kappa, \mu$ const.

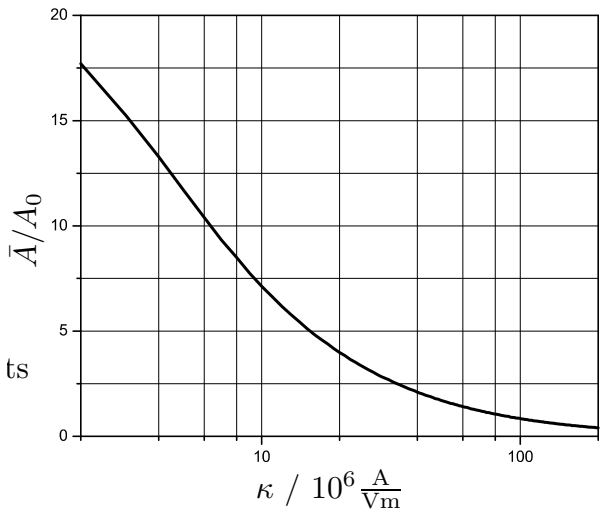

Fig. 6. $\bar{A} / A_{0}$ as a function of $\kappa, \omega, \mu$ const.

orthogonality of the cosine functions

$\int_{0}^{2 \pi} \cos (n \xi) \cos (p \xi) \mathrm{d} \xi= \begin{cases}0 & n \neq p \\ \pi & n=p \neq 0 \\ 2 \pi & n=p=0,\end{cases}$

we can isolate one coefficient $v_{\text {im }}$ on the left hand side of Eq. (4). Expanding the right hand side into a series of cosine functions by multiplication with $\cos \left(\alpha_{m} x_{c}\right)$ (or $\cos \left(\tilde{\alpha}_{m} y_{c}\right)$, respectivly) and computing its integral along one of the contour sections we get

$\frac{\mu_{a}}{\mu_{i}} v_{i m} d_{i m}=\sum_{i=1}^{4} \sum_{n=1}^{\infty}\left[\gamma_{i n m} v_{i n}\right]+b_{m}, \quad m \in \mathbb{N}$.

If we take only the first $N$ series elements into account, this equation can be written as a matrix equation

$\Gamma \mathbf{v}=\mathbf{b}$,

with matrix $\Gamma$ and vectors $\mathbf{v}, \mathbf{b}$ of finite dimensions. Equation (6) represents a system of linear equations from which the $4 N$ unknown coefficients $v_{i n}$ can be computed.

The values of the coefficients $\gamma_{i n m}, d_{i m}$ and $b_{m}$, needed for the solution of the matrix equation, can be obtained analytically (see Fichte et al., 2004). 


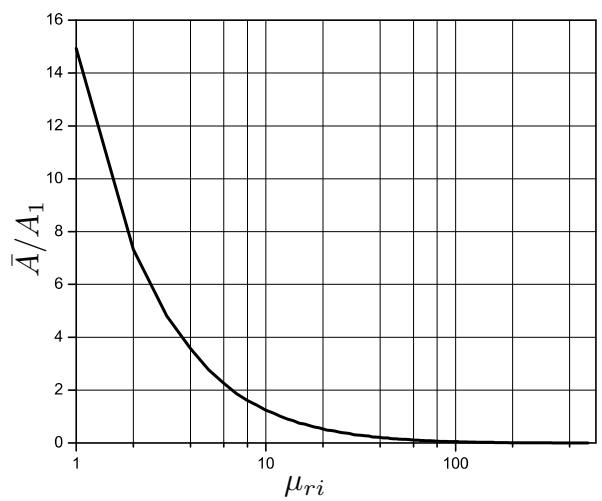

Fig. 7. $\bar{A} / A_{0}$ as a function of $\mu, \omega, \kappa$ const.

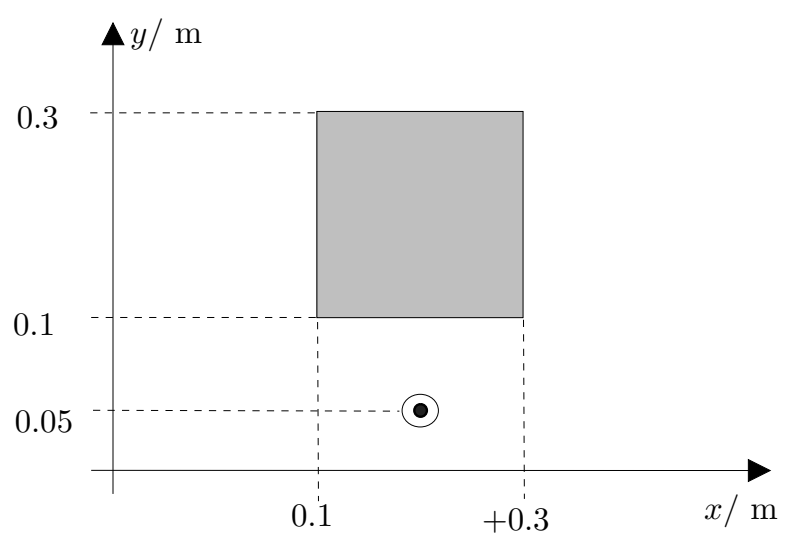

Fig. 8. Quadratic bar and single line.

Once the values of these coefficients are known, they can be used to express the vector potential outside the shielding bar as a series of known functions $A_{k}(x, y), k=1, \ldots, 4$ :

$A_{\mathrm{a}}(x, y)=\sum_{n=1}^{M} v_{1 n} A_{1}(x, y)+v_{2 n} A_{2}(x, y)+$

$+v_{3 n} A_{3}(x, y)+v_{4 n} A_{4}(x, y)$.

This solution for the vector potential $A_{\mathrm{a}}$ is used to determine the shielding properties of the bar by

$\Delta A=\frac{A_{\mathrm{a}}}{A_{\mathrm{e}}}$.

\section{Numerical results}

\subsection{Verification of method}

The applied method involves rarely used special functions, i.e. the exponential integral function for complex arguments, $E_{1}(z)$ (see Abramowitz and Stegun (1970), pp. 227 for details), which is not included in commericial numerical software packages. A focus of our paper is on the validation of the model. Therefore, in a first step, we use the presented method on a conducting bar which has been placed into an

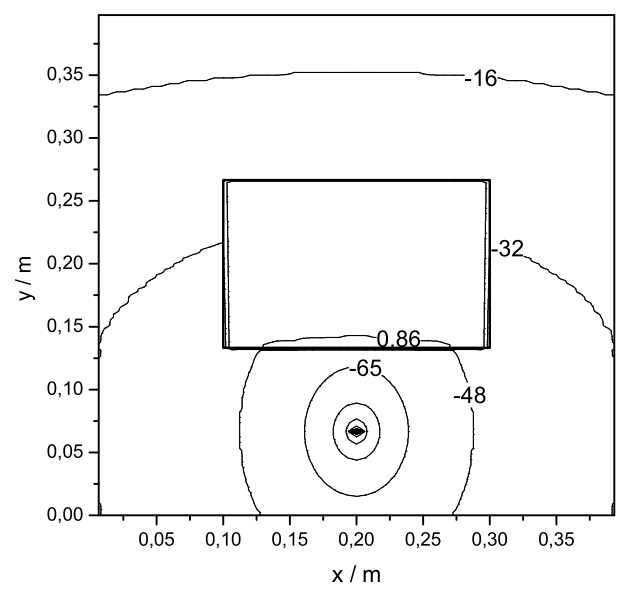

Fig. 9. Magnetic field at $50 \mathrm{~Hz}$.

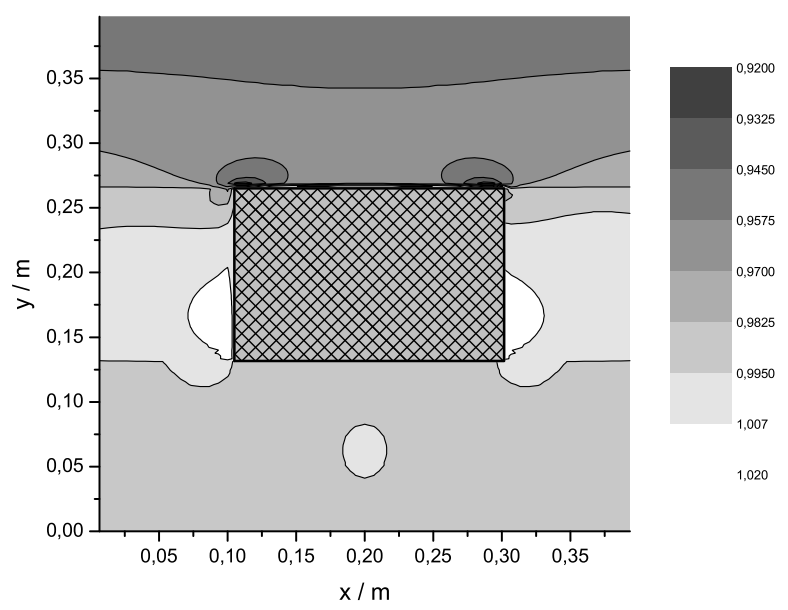

Fig. 10. Magnetic field at $50 \mathrm{~Hz}$.

homogenous magnetic field - see Fig. (3 - and compare the results with the expected behaviour.

The following Fig. 4 shows the absolute value of the modified vector potential inside the plate.

As an additional verification, different values of $\omega, \kappa$ and $\mu_{\mathrm{ri}}=\frac{\mu_{\mathrm{i}}}{\mu_{0}}$ are considered. We calculated the average of the absolute of $A_{\mathrm{i}}^{*}$ over the cross-section of the bar $\Omega$ as functions of . By this we can estimate which amount of the outside field permeates into the conducting area. The average of $A_{\mathrm{i}}^{*}$ has been normalized on the value $A_{0}=1 \cdot 10^{-6} \frac{V s}{A}$ in Figs. 5 and 6 and on $A_{1}=1 \cdot 10^{-7} \frac{V s}{A}$ in Fig. 7 .

\subsection{Single exciting line}

In a next step, one conducting bar of quadratic cross-section with $a=b=0,1 \mathrm{~m}$ is placed at $\boldsymbol{r}_{q}=(-0.1,-0.1) \mathrm{m}$. One single exciting line, carrying $I=100 \mathrm{~A}$, is placed at $(0.0$, $-0.15) \mathrm{m}$, as shown in Fig. 8.

Figure 9 displays the resulting lines of the magnetic field for $\omega=50 \frac{1}{\mathrm{~s}}$ and $\kappa=57 \cdot 10^{6} \frac{\mathrm{A}}{\mathrm{Vm}}, \mu_{\mathrm{ri}}=1$; the field attenuation 


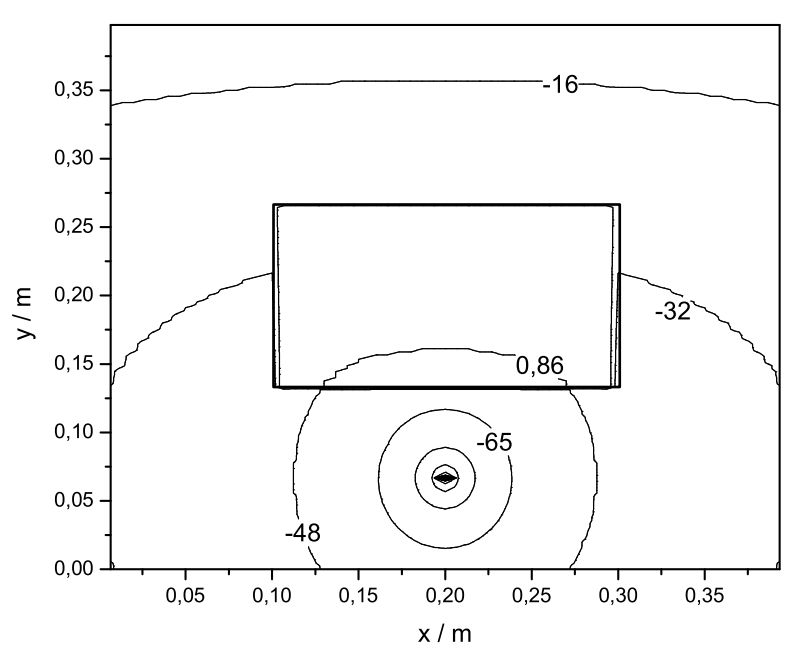

Fig. 11. Magnetic field at $5 \mathrm{~Hz}$.

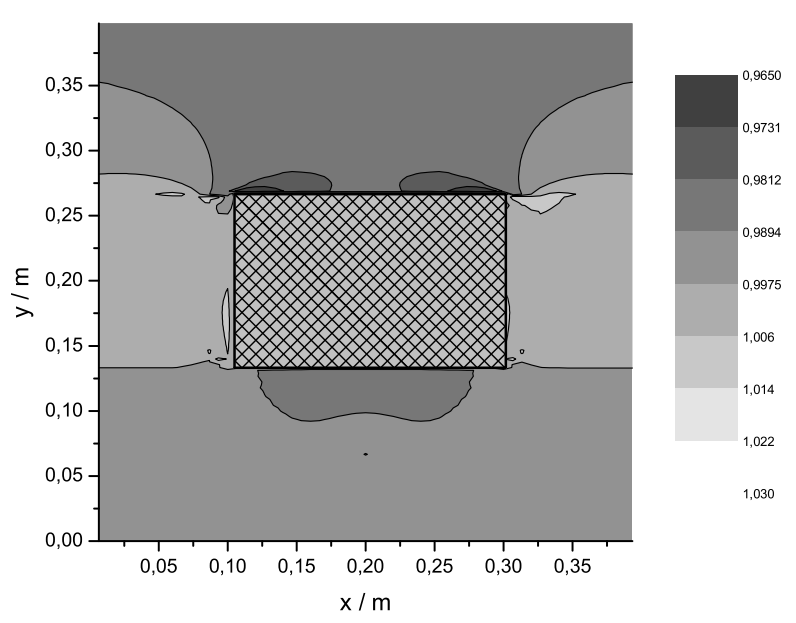

Fig. 12. Field attenuation at $5 \mathrm{~Hz}$.

$\Delta A_{\mathrm{a}}$ (and thereby the shielding effect of the conducting bar) is plotted in Fig. 10. The resulting plots are slightly nonsymmetric because of roundoff-errors.

Figures 11 and 12 show the lines and the attenuation of the magnetic field for the same configuration and a significantly lower frequency of $\omega=5 \frac{1}{\mathrm{~s}}$. Conductivity and permeability are $\kappa=57 \cdot 10^{6} \frac{\mathrm{A}}{\mathrm{Vm}}, \mu_{\text {ri }}=1$.

\section{Conclusions}

We applied a hybrid method to determine the field properties of a rectangular conducting bar in the field of an exciting loop. While Helmholtz' equation governing the vector potential inside the bar has been solved by separation, we obtained an integral representation for the vector potential in the exterior space from Green's second theorem. Coupling these two equations for points on the contour of the bar resulted in a system of linear equations. The solution to this system delivered the coefficients describing all fields. This method has been used to calculate the shielding properties of a conducting bar placed in the field of a exciting loop.

\section{References}

Abramowitz, M. and Stegun, I. A.: Handbook of Mathematical Functions, New York 1970.

Ehrich, M., Fichte, L. O. and Lüer, M.: Contribution to Boundary Integrals by the Singularity of Kernels satisfying Helmholtz' Equation CJMW'2000 China-Japan Joint Meeting on Microwaves, Nanjing, 2000.

Fichte, L. O., Ehrich, M., and Kurz, S.: An Analytical Solution to the Eddy Current Problem of a Conducting Bar EMC 2004 International Symposium on Electromagnetic Compatibility, Sendai Conference Proceedings CD-ROM, 2004.

Hanson, G. W. and Yakovlev, A. B.: Operator Theory for Electromagnetics, Springer, New York 2002. 